\title{
Wer ist ... \\ Hans-Joachim Schmoll?
}

Wie beginnen Sie Ihren Tag?

Schmoll: Viel zu oft nach einer zu kurzen Nacht, oder im Hotel mit einem letzten Blick in die Vortragsdias; aber immer gespannt darauf, was der neue Tag bietet, welche neuen Begegnungen, Erkenntnisse, Schönheiten und - hoffentlich gute - Erfahrungen.

\section{Was treibt Sie an?}

Schmoll: Das Leben ist spannend und voller interessanter Möglichkeiten, etwas Positives zu tun, besonders für (Krebs-)Kranke: wirklich hilfreiches zu entdecken, zu entwickeln, zu implementieren; das Leid der Krebskranken insgesamt und jedes uns anvertrauten Patienten verringern zu helfen; Visionen für die Zukunft zu entwickeln und umsetzten ... und auch entsprechend Spuren zu hinterlassen.

\section{Als Kind wollten Sie ...?}

Schmoll: Sein wie Albert Schweizer: Philantrop im ursprünglichen Sinne; ein Arzt, der sein Leben der Hilfe für leidende Menschen und Patienten widmet; Künstler und Musiker sein, der auf herrlichen Orgeln spielt; zugleich wollte ich Maler und Architekt sein.

\section{Was beeindruckt Sie an anderen Menschen?}

Schmoll: Ich bewundere Menschen, die ein großes Talent, Intelligenz, Kreativität und Schaffenskraft haben - diese Gaben aber nicht so sehr zum eigenen Wohl und Fortkommen einsetzen (was leider so oft der Fall ist), sondern ihre Kraft und Leidenschaft in den Dienst an anderen Menschen stellen.

\section{Worauf sind Sie besonders stolz?}

Schmoll: Ich hatte Glück als Anfänger in der Onkologie 1972, am ersten Tag Ifosfamid zur "Testung" bei Patienten mit refraktären Keimzelltumoren einsetzen zu können: diese todgeweihten jungen Männer hatten drama-

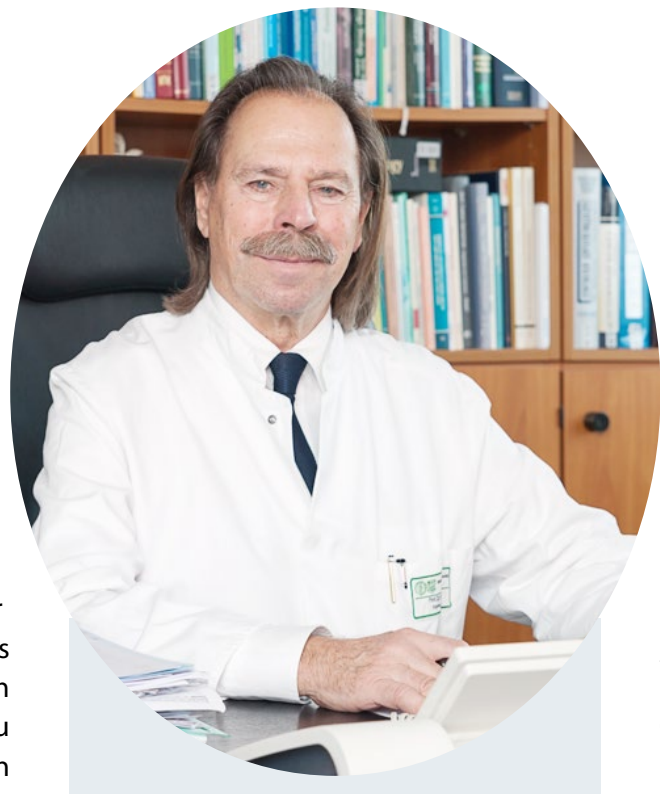

So wie der "Schmoll", das Kompendium Internistische Onkologie, ein Standardwerk ist, ist sein Herausgeber Prof. Dr. med. Hans-Joachim Schmoll eine Institution in der Medizinischen Onkologie. Denn er hat viel bewegt: Er hat z. B. die erste internationale Arbeitsgruppe "Hodentumoren" aufgebaut - mit der ersten internationalen Konsensusleitlinie, und später auch für kolorektale Karzinome mit den Konsensusleitlinien der European Society for Medical Oncology (ESMO). In Deutschland hat er etwa die Arbeitsgruppen in der Arbeitsgemeinschaft Internistische Onkologie (AIO) der Deutschen Krebsgesellschaft aufgebaut.

Seit 1995 ist Schmoll in Halle tätig und hat dort als Direktor die Klinik für Onkologie und Hämatologie sowie die Sachsen-Anhaltische Krebsgesellschaft aufgebaut. Seit 2014 leitet er die Arbeitsgruppe klinische Forschung in der Onkologie. Dass er auch für Neues aufgeschlossen ist, zeigt sich nicht nur daran, dass er in Halle das Landeszentrum für Zell- und Gentherapie aufgebaut hat. Sondern auch daran, dass die Neuauflage des Kompendiums auch als eBook herauskommen wird. tische Remissionen. Wir entwickelten in den nächsten Jahren parallel zu Lawrence Einhorn die Therapie zur Heilung dieser Patienten - ebenso wie viele weitere kleinere „Durchbrüche" bei anderen Tumoren. Stolz bin ich auch auf den Neuaufbau einer Klinik für Hämatologie und Onkologie an der Martin-Luther-Universität Halle, vor allem den Aufbau des Landeszentrums für Zell- und Gentherapie. Am wichtigsten aber war die Erstellung des "Kompendiums Internistische Onkologie" mit dem Ziel, die rasante Entwicklung in der Onkologie für den Anwender jedes Fachgebietes klar und systematisch darzulegen.

\section{Was möchten Sie noch erreichen?}

Schmoll: Gerade sitze ich an der 5. Auflage des "Kompendiums". Neben der Druckversion wird zugleich ein ständig zu erneuerndes eBook veröffentlicht. Sorgen macht mir die Struktur der onkologischen Versorgung und Ausbildung im Land: Wo sind die - in fast allen anderen Ländern vorhandenen - „Multidisziplinären Cancer Centers", wo ausschließlich Krebsspezialisten für jede Tumorart und Fachdisziplin die modernste und beste "passgenaue" Therapie bereithalten?

\section{Was war Ihre wichtigste Entscheidung?}

Schmoll: Arzt und Onkologe an einer forschenden Institution zu werden.

\section{Wie halten Sie Balance in Ihrem Leben?}

Schmoll: Täglich 0,5-1 Stunde "meditatives Innehalten" gibt Kraft und ordnet den Kopf.

\section{Wie beenden Sie Ihren Tag?}

Schmoll: Am liebsten mit allem, was sonst nicht geht, z. B. Musik hören, dazu ein kühles Weißbier oder Wein von der Saale - auch wenn die Zeit meist nur letzteres ermöglicht. 